\title{
When Should the Internal Iliac Arteries be Preserved in Patients Undergoing Endovascular or Open Repair of Aortoiliac Aneurysms?
}

\author{
MANISH MEHTA, M.D., ${ }^{1}$ FRANK J. VEITH, M.D., ${ }^{1}$ \\ and TAKAO OHKI, M.D. ${ }^{1}$
}

ABSTRACT Internal iliac artery (IIA) occlusion during aortic aneurysm repair has been associated with considerable morbidity. We analyzed the consequences of interrupting one or both IIAs in the standard surgical or endovascular treatment of aortoiliac aneurysms (AIAs). From 1992 to 2000, 158 patients with abdominal aortic aneurysms $(n=66)$, iliac aneurysms $(n=28)$, or AIAs $(n=64)$ required interruption of one $(n=134)$ or both $(n=24)$ IIAs as part of their endovascular $(n=110)$ or open repair $(n=48)$. Endovascular treatment was performed with a variety of industry- or surgeonmade grafts in combination with coil embolization of the IIAs. The standard surgical techniques included oversewing or excluding the origins of the IIAs, and extending the prosthetic graft to the external iliac or femoral artery. There were no cases of buttock necrosis, ischemic colitis requiring laparotomy, or death when one or both IIAs were interrupted. Persistent buttock claudication occurred after $16(12 \%)$ of the unilateral and $3(13 \%)$ of the bilateral IIA interruptions. Impotence occurred in $7(9 \%)$ of the unilateral and $2(11 \%)$ of the bilateral IIA interruptions. Minor neurological deficits of the lower extremity were observed in $2(1.5 \%)$ of the patients with unilateral IIA interruption. Although IIA flow should be preserved if possible, selective interruption of one or both IIAs can usually be accomplished safely during endovascular and open repair of anatomically challenging AIAs. We believe other comorbid factors,

Perspectives in Vascular Surgery and Endovascular Therapy, Volume 14, Number 1, 2001. Address for correspondence and reprint requests: Frank J. Veith, M.D., Division of Vascular Surgery, Montefiore Medical Center, 111 E. 210th Street, Bronx, New York 10467. 1Division of Vascular Surgery, Montefiore Medical Center, 111 E. 210th Street, Bronx, New York 10467. ${ }^{1}$ Department of Surgery, Division of Vascular Surgery, Montefiore Medical Center, Bronx, NY. Copyright (C) 2001 by Thieme Medical Publishers, Inc., 333 Seventh Avenue, New York, NY 10001, USA. Tel: +1(212) 584-4662. 1531-0035,p;2001,14,01,017,028,ftx,en;00105x. 
such as shock, distal embolization, or the failure to preserve collateral branches from the external iliac and femoral arteries, may have contributed to the morbidity in other reports of IIA interruption.

Keywords Internal iliac artery, aortoiliac aneurysm, pelvic ischemia

Approximately $20 \%$ of patients presenting with abdominal aortic aneurysms have concomitant iliac artery aneurysms. ${ }^{1}$ In many of these the distal common iliac artery is aneurysmal, and in some instances interruption of one or both internal iliac arteries (IIAs) in conjunction with aortoiliac or aortofemoral bypass is needed to exclude the aneurysm. Although several studies in the obstetrics and gynecology, ${ }^{2}$ urology, ${ }^{3}$ and trauma ${ }^{4}$ literature suggest that IIAs can be interrupted without any adverse sequelae, the importance of preserving one or both of these vessels during repair of aortoiliac aneurysms (AIAs) is stressed in the vascular surgery literature. IIA interruption, particularly bilateral, during open aortoiliac aneurysm repair has been reported to be associated with considerable morbidity, including buttock necrosis, severe lower extremity neurological deficits, ischemic colitis, impotence, and buttock claudication. ${ }^{5-9}$ The advent of endovascular aneurysm repair has rekindled interest in IIA interruption because of the frequent lack of adequate distal fixation sites in the aneurysmal common iliac arteries. Current endovascular grafts must therefore often be fixed in the external iliac arteries (EIAs). This requires exclusion of one or both IIAs and prompted us to reevaluate the risks and consequences of IIA interruption. In this article, we review the morbidity of unilateral and bilateral IIA interruption in patients undergoing either standard open surgical or endovascular AIA repair.

\section{MONTEFIORE EXPERIENCE WITH IIA INTERRUPTION}

\section{Patients}

All patients who had abdominal aortic aneurysms larger than $5 \mathrm{~cm}$ and iliac artery aneurysms larger than $3.5 \mathrm{~cm}$, diagnosed between November 1992 and March 2000, were eligible for standard open surgical or endovascular repair. High-risk patients with severe cardiopulmonary disease, a hostile abdomen, or other major coexisting medical problems were offered treatment with different types of endovascular grafts. These techniques were also offered to some better-risk patients when protocols became available. Patients who did not meet the inclusion criteria for endovascular repair or who refused such treatment were managed by standard open surgical repair. Charts, perioperative arteriograms, computed tomography (CT) scans, and operative reports were reviewed and the aortoiliac vascular anatomy deter- 
mined for each patient. Postoperatively, all patients underwent a thorough evaluation to elicit signs and symptoms of buttock claudication and neurological deficits. Impotence was evaluated by privately questioning all men regarding their ability to have an erection preoperatively as well as postoperatively. When information on buttock claudication and impotence was unavailable in charts, patients were contacted by telephone.

\section{Procedures}

Our techniques for open and endovascular aneurysm repairs have been described elsewhere. ${ }^{10,11}$ In standard surgical repairs, the proximal anastomosis was constructed in an end-to-end fashion just distal to the renal vein. The location of the distal anastomosis varied depending on the extent of the aneurysm. In endovascular repairs, the endografts were fixed proximally just below the renal arteries for aortic aneurysms, and in the proximal common iliac artery for iliac artery aneurysms with defined necks $>1.5 \mathrm{~cm}$ in length. Distal fixation sites were either the external iliac or the common femoral artery.

For patients with AIAs $(n=88)$ endovascular repair was performed with a variety of industry-made (EVT-Ancure: Guidant Endovascular Tech., Menlo Park, CA; Talent: Medtronic, Sunrise, FL; AneuRx: Medtronic, Sunrise, FL; Vanguard: Boston Scientific, Natick, MA; Gore-Excluder: WL Gore, Flagstaff, AZ; Cook-Xenith) and surgeon-made grafts. ${ }^{11}$ Due to unsuitable anatomy or unavailability of industry-made bifurcated stent grafts, patients with an AAA $(n=66)$ that were considered high risk due to severe cardiopulmonary disease, a hostile abdomen, or other major coexisting medical problems underwent endovascular repair with surgeon-made grafts. This repair included an aortounifemoral stent graft combined with a femorofemoral bypass, coil embolization of the ipsilateral HA, and placement of an occluder device in the contralateral common iliac artery. Of these patients, 64 (97\%) had unilateral and $2(3 \%)$ had bilateral HA interruptions.

\section{Techniques of IIA Interruption}

In standard surgical repairs, when extensive common iliac or IIA aneurysms were encountered, the orifice of the common iliac artery was oversewn from within. However, occasionally the IIAs were ligated when it was technically feasible. The EIA was ligated or oversewn proximally, and the distal anastomosis constructed to the distal external iliac or the common femoral artery.

Depending on the diameter of the IIAs, Gianturco coils (Cook, Bloomington, IN) ranging between 5 and $10 \mathrm{~mm}$ were used to exclude flow to the IIAs, either preoperatively or intraoperatively. Coils were placed in the proximal IIA $(n=128)$ if it was not aneurysmal. If the IIA was aneurysmal $(n=4)$ 
the coils were placed in the nonaneurysmal branches. Flow interruption to both IIAs was staged in $8(73 \%)$ patients undergoing endovascular repair and $5(38 \%)$ patients undergoing open surgical repair. The ipsilateral IIA was coil embolized at the time of a preoperative arteriography, and the contralateral IIA was interrupted at the time of aneurysm repair. In four cases such embolization was impossible due to iliac artery tortuosity, and the origin of the IIA was simply covered with the endoluminal graft.

\section{RESULTS}

One hundred and fifty-eight patients with isolated abdominal aortic aneurysms $(n=66)$, iliac aneurysms $(n=28)$, or aortoiliac aneurysms $(n=64)$, underwent interruption of one $(n=134)$ or both $(n=24)$ IIAs as part of their endovascular $(n=110)$ or open $(n=48)$ treatment. The average age was 76.2 years, and comorbidities included coronary artery disease (59\%), hypertension $(61 \%)$, and severe chronic obstructive pulmonary disease $(22 \%)$ (Table 1). The mean aortic, common iliac, and IIA aneurysm diameters were $6.2,4.2$, and $4.7 \mathrm{~cm}$, respectively.

Reconstructions included aortobiiliac (common or external) in 61, aortobifemoral in 3, unilateral aortofemoral with a femorofemoral bypass and contralateral iliac artery interruption in 70, and unilateral iliofemoral in 24 patients. In these patients the IIA flow was interrupted either by preoperative coil embolization $(n=64)$, intraoperative coil embolization $(n=66)$, intraoperative endovascular-graft coverage $(n=4)$, or intraoperative ligation or oversewing $(n=48)$.

Postoperative morbidity included myocardial infarction, renal insufficiency, pneumonia, multisystem organ failure, and graft occlusion (Table 2). In no instance did interruption of the IIAs result in perineal necrosis, ischemic colitis requiring laparotomy, or death. Twenty-five percent of the uni-

Table 1 Patient Demographics

\begin{tabular}{|c|c|c|c|c|}
\hline IIA Interruption & Unilateral & & Bilateral & \\
\hline Type of repair & Endovascular & Open & Endovascular & Open \\
\hline Number of patients & 99 & 35 & 11 & 13 \\
\hline Male & $85(85 \%)$ & $28(80 \%)$ & $10(91 \%)$ & $13(100 \%)$ \\
\hline Female & $14(14 \%)$ & $6(17 \%)$ & $1(9 \%)$ & 0 \\
\hline $\mathrm{CAD}$ & $60(60 \%)$ & $19(54 \%)$ & $7(64 \%)$ & $7(54 \%)$ \\
\hline HTN61 (61\%) & $23(66 \%)$ & $6(55 \%)$ & $7(54 \%)$ & \\
\hline COPD & $24(24 \%)$ & $6(17 \%)$ & $5(50 \%)$ & 0 \\
\hline
\end{tabular}

CAD: Coronary artery disease

HTN: Hypertension

COPD: Chronic obstructive pulmonary disease 
Table 2 Postoperative Morbidity

\begin{tabular}{lllll}
\hline IIA Interruption & Unilateral & \multicolumn{3}{l}{ Bilateral } \\
\hline Type of repair & Endovascular & Open & Endovascular & Open \\
Number of patients & 99 & 35 & 11 & 13 \\
Myocardial infarction & $5(5 \%)$ & $1(3 \%)$ & 0 & 0 \\
Renal insufficiency & $9(9 \%)$ & $3(9 \%)$ & 0 & $1(8 \%)$ \\
Pneumonia & $2(2 \%)$ & $4(11 \%)$ & 0 & $3(23 \%)$ \\
Multisystem organ failure & $4(4 \%)$ & 0 & 0 & 0 \\
Graft occlusion & $4(4 \%)$ & 0 & $1(9 \%)$ & 0 \\
& & & & \\
\hline
\end{tabular}

lateral and $21 \%$ of the bilateral IIA interruption patients were impotent preoperatively. Of the remaining, new onset impotence resulted in only 7 (9\%) of the unilateral and only $2(11 \%)$ of the bilateral IIA interruptions. Neurological deficits were only seen in the unilateral IIA interruption group: One patient developed foot drop, and another had minor bilateral lower extremity weakness (Table 3). Although buttock claudication developed in 48 (36\%) of the unilateral and $11(46 \%)$ of the bilateral IIA interruptions within the first month, these symptoms persisted in only $16(12 \%)$ of the unilateral and $3(13 \%)$ of the bilateral IIA interruptions at 1 year (Table 4$)$. Nine patients $(6.0 \%)$ died secondary to myocardial infarction and multisystem organ failure.

\section{DISCUSSION}

IIA interruption, particularly bilateral, has been considered a morbid procedure. Several reports have suggested that acute interruption of IIAs during AIA repair is a likely cause of buttock necrosis, spinal cord ischemia, and isch-

Table 3 Complications of Hypogastric Artery Interruptions

\begin{tabular}{lllll}
\hline IIA Interruption & Unilateral & & Bilateral \\
\hline & Endovascular & Open & Endovascular & Open \\
Type of repair & $85 / 14$ & $28 / 6$ & $10 / 1$ & $13 / 0$ \\
Number of patients (male/female) & 0 & 0 & 0 & 0 \\
Buttock necrosis & $2(2 \%)$ & $2(6 \%)$ & 0 & 0 \\
Ischemic colitis (mucosal) & $2(2 \%)$ & 0 & 0 & 0 \\
Minor neurological deficits & $10(10 \%)$ & 0 & $2(18 \%)$ & $1(8 \%)$ \\
Buttock claudication (<2 block) & $6(6 \%)$ & 0 & 0 & 0 \\
Buttock claudication (>2 block) & $6(9 \%)$ & $1(5 \%)$ & $1(9 \%)$ & $1(8 \%)$ \\
Impotence (new onset)* & & & & \\
\hline
\end{tabular}

*Preoperative impotence: unilateral (25\%) and bilateral (21\%) HA interruption. 
Table 4 Improvement in the Incidence of Buttock Claudication

\begin{tabular}{lccc}
\hline & IIA Interruption & Claudication @ I month & Claudication @ I year \\
\hline Unilateral & 134 & $48(36 \%)$ & $16(12 \%)$ \\
Bilateral & 24 & $11(46 \%)$ & $3(13 \%)$ \\
& & & \\
\hline
\end{tabular}

emic colitis with a considerable mortality. ${ }^{12-14}$ Therefore, unilateral and especially bilateral IIA interruption should be and was avoided during open as well as endovascular AIA repair, and we concur with this premise if preservation of IIA flow was possible. However, this may be difficult or impossible in some instances when AIAs involve the iliac bifurcation or the IIAs. In such instances our results suggest that unilateral and bilateral IIA interruption during AIA repair is a relatively innocuous procedure. This appears to be true particularly when IIAs are interrupted at their origin, the collateral branches are preserved, and comorbid factors such as shock and distal embolization do not occur.

When aortoiliac aneurysms extend to the iliac bifurcation or involve the IIAs, it may be difficult or impossible to preserve flow to one or both IIAs. During open surgical repair, anatomic limitations may preclude revascularization of the IIAs because this may require extensive pelvic dissection in a relatively inaccessible area in which the arterial anatomy is distorted, and injuries to large veins may be hard to avoid. Furthermore, currently available endovascular grafts lack the versatility to be fixed distally into aneurysmal iliac arteries safely and must sometimes be extended to the external iliac arteries. In these instances, interruption of the IIA is required for complete exclusion of the aortoiliac or iliac aneurysms (Fig. 1).

When possible, the IIAs are interrupted at their origin by oversewing, coil embolization, or stent-graft coverage. This preserves the extensive network of collateral connections between branches of the IIA, the EIA, and the femoral arteries (Fig. 2). Iliopoulos ${ }^{15}$ examined acute pressure changes of the pelvic circulation with IIA interruption and showed that branches of the ipsilateral EIA and femoral arteries provide a more significant collateral pathway than the contralateral IIA. ${ }^{15}$ We routinely try to preserve the iliac and femoral circumflex branches, particularly when interrupting one or both IIAs.

In 1986, Picone et al. ${ }^{12}$ reported their experience with 7 patients who had complications of IIA interruption during abdominal aortic surgery. They suggested that the most likely cause of buttock necrosis, ischemic colitis, and neurological deficits was acute interruption of flow to the IIAs. Although these authors reported a mortality of $70 \%$ with bilateral IIA interruptions, $43 \%$ of their patients had had urgent or emergent operations. Furthermore, two-thirds of the patients experienced perioperative hypotension and throm- 


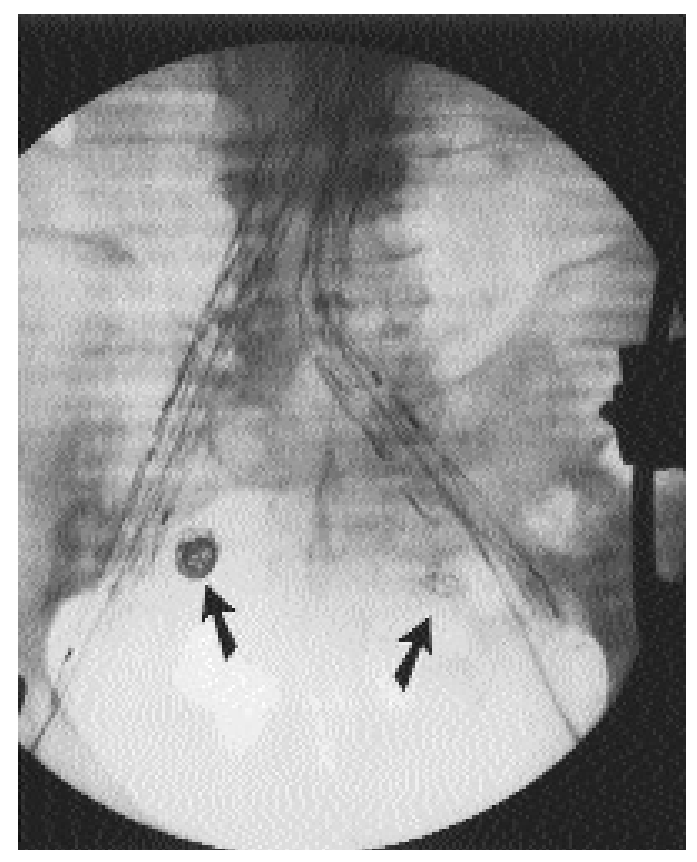

Fig. 1 Coil embolization of the IIAs (arrows) and bilateral iliac limb extensions to the EIA for complete exclusion of the AIA. (From Mehta M, Veith FJ, Ohki T, et al: JVS 2001;33:527-532.)

bosis. In 1987, Iliopoulos et al. ${ }^{13}$ reported a similar experience with complications after flow interruption to the IIAs. However, two-thirds of their patients had also experienced perioperative hypotension, distal emboli, or thrombosis. The latter may lead to impaired pelvic collateral flow, which may be vital in the setting of unilateral and bilateral IIA interruption. Our experience includes elective cases in which perioperative hemodynamic instability, distal emboli, and thrombosis were encountered far less frequently than in previously published reports documenting a dismal outcome following bilateral IIA interruption.

None of our patients with unilateral or bilateral IIA interruption developed buttock necrosis or major neurological deficits. Only four patients with unilateral, and surprisingly none with bilateral, IIA interruption developed symptoms of ischemic colitis. These symptomatic patients underwent colonoscopy, revealing some mucosal ischemic colitis. In all patients these symptoms resolved without the need for laparotomy or bowel resection. Over the past 8 years we have operated on 435 patients with an AAA without flow interruption to either IIA. Only 7 developed ischemic colitis, and a similar incidence has been well documented by other authors as well. 16,17 One can speculate that perhaps embolization and thrombosis of branches of the IIAs, in addition to the interruption of flow to the inferior mesenteric artery, may lead to these serious complications. It is also possible that unrec- 

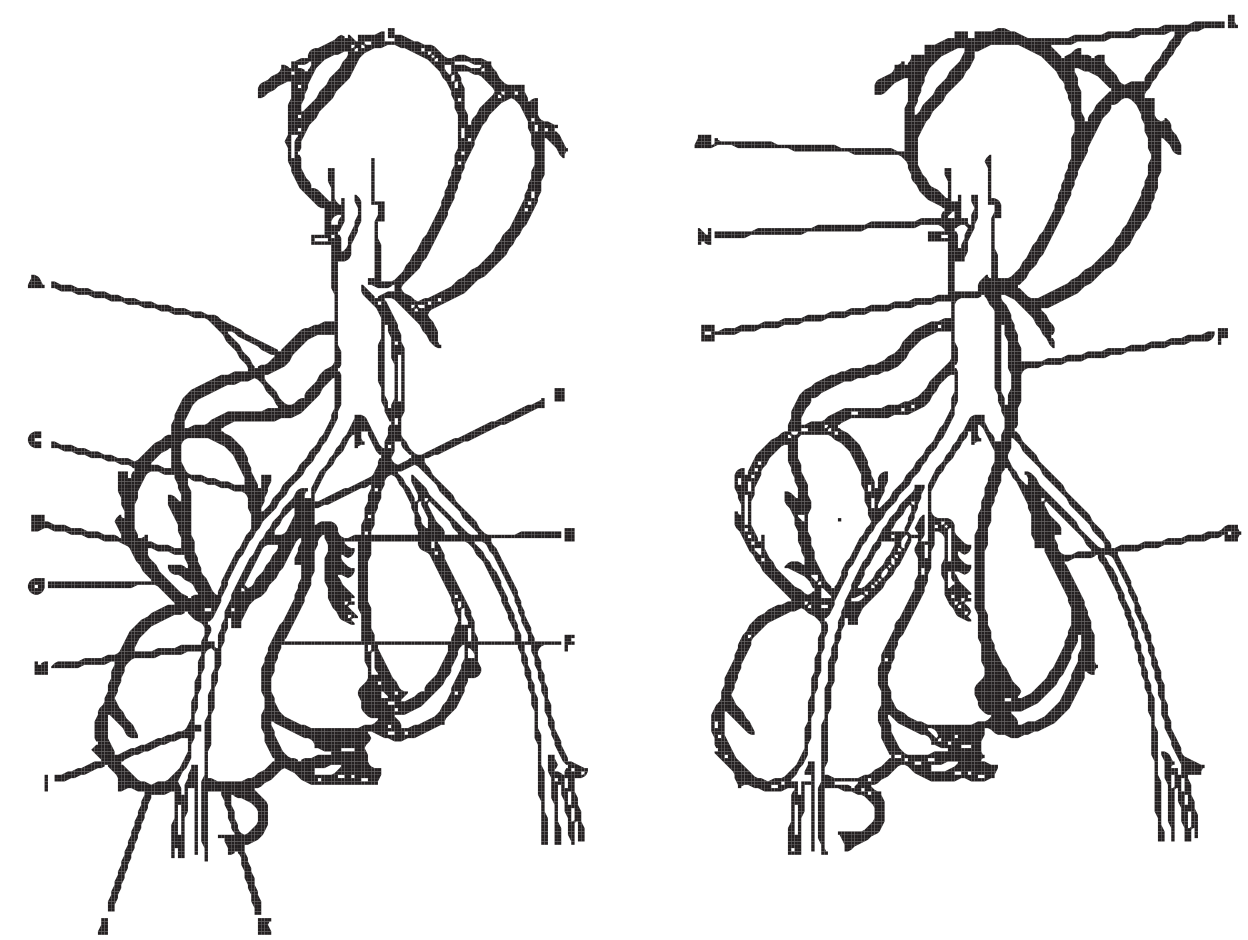

Fig. 2 Pelvic collateral circulation. (A) Lumbar arteries. (B) Internal iliac artery. (C) Superior gluteal artery. (D) Iliolumbar artery, branch of inferior gluteal artery. (E) Lateral sacral arteries. (F) Obturator artery. (G) Deep iliac circumflex artery. (H) External iliac artery. (I) Common femoral artery. (J) Lateral femoral circumflex artery. (K) Medial femoral circumflex artery. (L) Marginal artery of Drummond. (M) Middle colic artery. (N) Superior mesenteric artery. (O) Inferior mesenteric artery. (P) Superior rectal artery. (Q) Middle and inferior rectal arteries. (From Mehta M, Veith FJ, Ohki T, et al: JVS 2001;33:527-532.)

ognized celiac and superior mesenteric artery stenoses may play a role in these complications. Although the celiac and superior mesenteric arteries were patent in all patients, lateral views on their arteriograms were not always available, making it difficult to evaluate the presence of stenosis. Preoperatively the inferior mesenteric artery (IMA) was patent in $27(25 \%)$ of the endovascular and 11 (23\%) of the open surgical patients. Of these, the IMA was reimplanted in only one patient who underwent an open AIA repair with bilateral IIA interruption. The incidence of colonic ischemia, buttock claudication, and new onset impotence was no different in this group when compared with patients with preoperative IMA occlusion.

Buttock claudication, when it occurred, generally improved within months. In the first postoperative month, 36\% of the unilateral and $46 \%$ of the bilateral IIA interruption patients developed buttock claudication. One 
year postoperatively, only $12 \%$ of the unilateral and $13 \%$ of the bilateral IIA interruption patients complained of buttock claudication. A significant $(P<0.05)$ difference in the incidence of claudication was noted between those who had endovascular interruption (16\%), and those that had surgical interruption $(3 \%)$ of flow to the IIAs. Although coils are always placed proximally in the IIA, they can embolize distally into secondary branches and interrupt the pelvic collateral circulation. Although we did not analyze the exact location of coils in the IIAs and its relation to morbidity, Cynamon et al. ${ }^{18}$ have recently published their experience with 13 patients who developed buttock claudication after IIA coil embolization. They reported a substantial difference in the incidence of buttock claudication when coils were placed in the proximal IIA (10\%) as opposed to its distal branches (55\%). New onset of impotence occurred in $9 \%$ of the unilateral and $11 \%$ of the bilateral IIA interruptions and was not considered to be a substantial problem in our patient population.

In the postoperative period, 9 patients died due to myocardial infarction and multisystem organ failure. None of these patients developed buttock necrosis, ischemic colitis, lower extremity neurological deficits, or rhabdomyolysis. Autopsies were performed on only three patients, none revealing histologic findings consistent with ischemic colitis or muscle necrosis. Although we cannot be certain, it is our opinion after a careful analysis of the clinical course in the remaining patients that the IIA interruption did not contribute to the cause of death in any of these patients.

Our results suggest that unilateral and bilateral IIA interruption is a relatively safe procedure, particularly when pelvic collateral circulation from the external iliac and femoral arteries is preserved. These findings are supported by two other recent papers on IIA interruption with endovascular repair of AIAs. ${ }^{19,20}$ Although these studies have smaller numbers and only include endovascular aneurysm repair, they support our finding that patients do not experience life-threatening complications after unilateral and bilateral IIA interruption. However, the issue of bilateral IIA interruption remains controversial. Parodi reported on reimplantation of the IIA into the distal EIA to facilitate endoluminal treatment of extensive AIAs and to avoid interruption of bilateral IIAs. ${ }^{21}$ Whenever possible, we attempt to save one or both IIAs and have even reimplanted the IIA into the EIA (Fig. 3). However, we have found this to be a cumbersome procedure, particularly in patients who are obese or have calcified iliac arteries.

We have been asked to answer the following question: "When should the IIAs be preserved in patients undergoing endovascular or open repair of AIAs?" We strive to preserve flow to the IIAs whenever possible to avoid not only the potential for persistent buttock claudication and impotence but also some of the more serious sequelae reported by others. However, in the treatment of extensive AIAs, when preservation of flow to the IIA is not possible, our experience suggests that interruption of one or both IIAs can be performed relatively safely without life-threatening complications. 

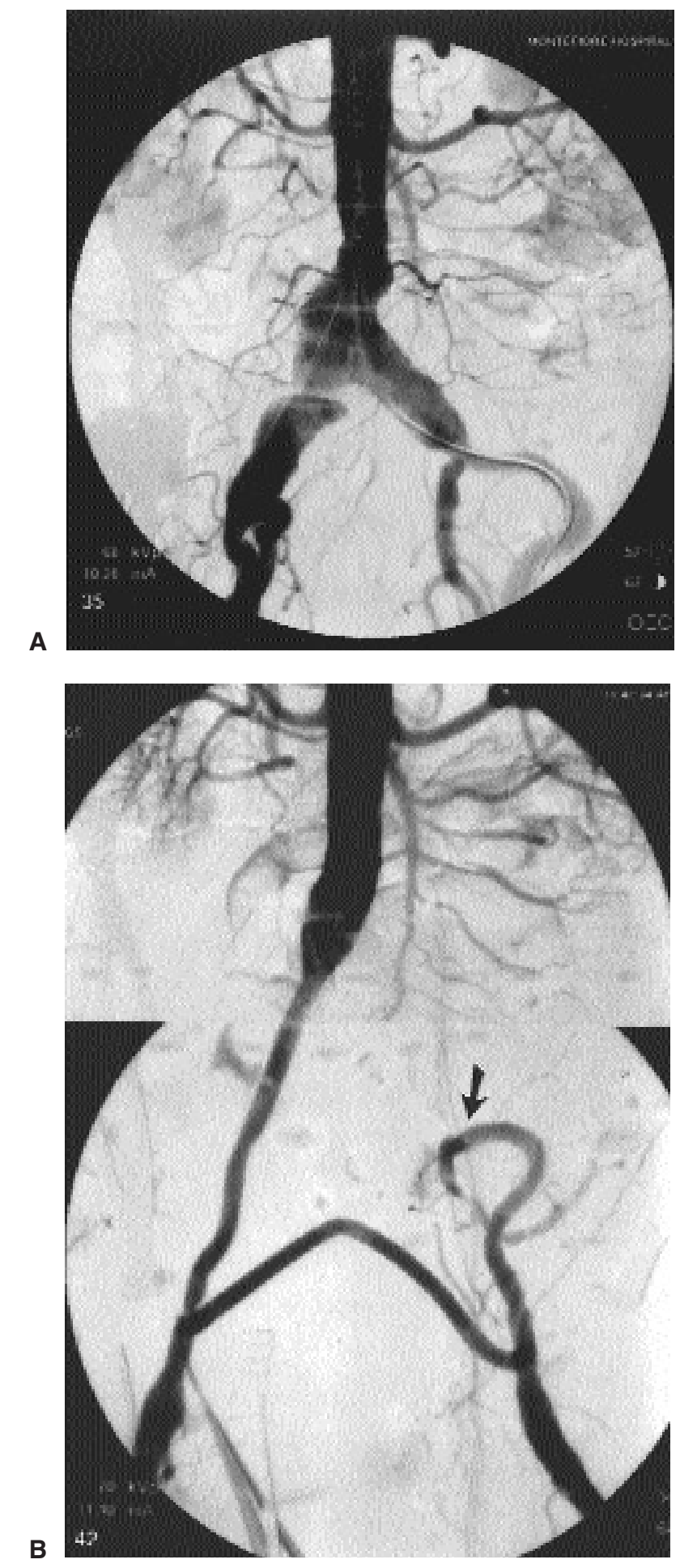

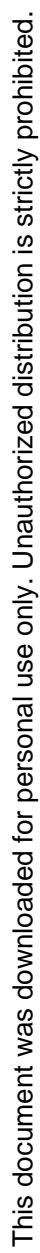

Fig. 3 (A) Arteriogram of aortoiliac aneurysm extending to the iliac bifurcation bilaterally. (B) Aorto-uni-femoral stent graft (Montefiore endovascular graft) and right to left fem-femoral bypass graft (PTFE). Flow to the left IIA is preserved through an end-to-end anastomosis between the EIA-IIA (arrow). (From Mehta M, Veith FJ, Ohki T, et al: JVS 2001;33:527-532.) 


\section{REFERENCES}

1. Armon MP, Wenham PW, Whitaker SC, et al. Common iliac artery aneurysms in patients with abdominal aortic aneurysms. Eur J Vasc Endovasc Surg 1998;15(3):255-257

2. Clark SL, Phelan JP, Yeh SY, et al. Hypogastric artery ligation for obstetric hemorrhage. Obstet Gynecol 1985;66(3):353-356

3. Bao ZM. Ligation of the internal iliac arteries in 110 cases as a hemostatic procedure during suprapubic prostatectomy [letter]. J Urol 1980;124(4):578

4. Agolini SF, Shah K, Jaffe J, et al. Arterial embolization is a rapid and effective technique for controlling pelvic fracture hemorrhage [see comments]. J Trauma 1997;43(3): 395-399

5. Andriole GL, Sugarbaker PH. Perineal and bladder necrosis following bilateral internal iliac artery ligation. Report of a case. Dis Colon Rectum. 1985;28(3):183-184

6. Senapati A, Browse NL. Gluteal necrosis and paraplegia following postoperative bilateral internal iliac artery occlusion. J Cardiovasc Surg (Torino) 1990;31(2):194-196

7. Gloviczki P, Cross SA, Stanson AW, et al. Ischemic injury to the spinal cord or lumbosacral plexus after aorto-iliac reconstruction. Am J Surg 1991;162(2):131-136

8. Cikrit DF, O'Donnell DM, Dalsing MC, et al. Clinical implications of combined hypogastric and profunda femoral artery occlusion. Am J Surg 1991;162(2):137-140; discussion 140-141

9. Paty PK, Shah DM, Chang BB, et al. Pelvic ischemia following aortoiliac reconstruction. Ann Vasc Surg 1994;8(2):204-206

10. Veith FJ, Hobson RW, Williams RA, Wilson SE. Vascular Surgery, Principles and Practice, 2nd ed., Vol. 1. New York: McGraw-Hill; 1994:1156-1173

11. Ohki T, Veith FJ, Sanchez LA, et al. Varying strategies and devices for endovascular repair of abdominal aortic aneurysms. Semin Vasc Surg 1997;10(4):242-256

12. Picone AL, Green RM, Ricotta JR, et al. Spinal cord ischemia following operations on the abdominal aorta. J Vasc Surg 1986;3(1):94-103

13. Iliopoulos JI, Howanitz PE, Pierce GE, et al. The critical hypogastric circulation. Am J Surg 1987;154(6):671-675

14. Kalliafas S, Albertini JN, Macierewicz J, et al. Incidence and treatment of intraoperative technical problems during endovascular repair of complex abdominal aortic aneurysms. J Vasc Surg 2000;31:1185-1192

15. Iliopoulos JI, Hermreck AS, Thomas JH, Pierce GE. Hemodynamics of the hypogastric arterial circulation. J Vasc Surg 1989;9(5):637-641; discussion 641-642

16. Zelenock GB, Strodel WE, Knol JA, et al. A prospective study of clinically and endoscopically documented colonic ischemia in 100 patients undergoing aortic reconstructive surgery with aggressive colonic and direct pelvic revascularization, compared with historic controls. Surgery 1989;106(4):771-779; discussion 779-780

17. Johnston KW. Multicenter prospective study of nonruptured abdominal aortic aneurysm. Part II. Variables predicting morbidity and mortality. J Vasc Surg 1989;9(3):437-447

18. Cynamon J, Lerer D, Veith FJ, et al. Hypogastric artery coil embolization prior to endoluminal repair of aneurysms and fistulas: buttock claudication, a recognizable but possibly preventable complication. J Vasc Interv Radiol 2000;11(50):573-577

19. Karch LA, Hodgson KJ, Mattos MA, et al. Adverse consequences of internal iliac artery occlusion during endovascular repair of abdominal aortic aneurysms. J Vasc Surg 2000;32:676-683

20. Criado FJ, Wilson EP, Valazquez OC, et al. Safety of coil embolization of the internal iliac artery in endovascular grafting of abdominal aortic aneurysms. J Vasc Surg 2000;32: 684-688

21. Parodi JC. Relocation of iliac artery bifurcation to facilitate endoluminal treatment of AAA. J Endovasc Surg 1999;6:342-347 
